\title{
THE ETIOLOGY OF FEMORAL EPIPHYSIAL SLIPPING
}

\author{
Colin Alexander, Auckland, New Zealand \\ From the Auckland Public Hospital
}

The object of this paper is to investigate a hypothesis on the cause of slipped femoral epiphysis.

Current etiological theories-Key (1926) suggested that epiphysiolysis was caused by periosteal weakening. Waldenström (1940) postulated a hormone disturbance inhibiting osteogenesis, and Howorth suggested synovitis (1941), an infection (1952), or osteochondrosis related to a vascular disturbance (1957). Evidence to support a possible endccrine basis was advanced by Harris (1950) and supported by Löfgren (1953) and Theander (1962). Toxic changes caused by the ingestion of aminonitriles have been suggested by Ponseti and McClintock (1956), Ponseti (1957), Selye and Ventura (1957), Andrén and Borgström (1958). Dulligan (1953) postulated muscle imbalance. Billing (1954) and Rennie (1960) suggested that deficient osteogenesis posteriorly producing obliquity of the cartilage plate was the main factor. Rennie considered that the horizontal force generated by the standing posture caused the deficient osteogenesis.
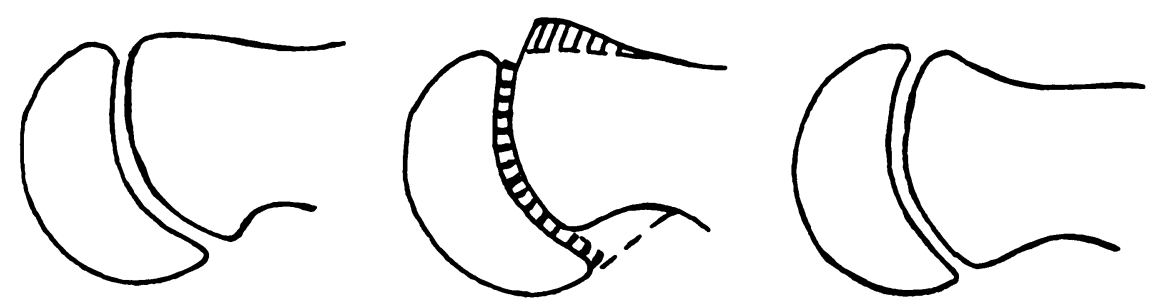

FIG. 1

Moulding of the femoral neck after displacement of the epiphysis. This is the mechanism suggested by Billing whereby both the shertening of the neck posteriorly and the altered plate angle can arise from epiphysial displacement. This change can be seen in Figures 2 and 3.

Backman (1957) supported Billings' concept, but in 1959 Billing and Severin showed that the short posterior neck found in the condition was the result rather than the cause of the slipping (Fig. 1). Michele (1960) postulated excessive iliopsoas traction during growth, and several writers have put the blame on trauma or on excessive static stress due to obesity.

None of these theories explains all the known facts. The normal state of other epiphyses (Burrows 1957) and the existence of unilateral cases make it difficult to assign more than an adjuvant role to the endocrine imbalance suggested by Harris. The theories implicating stress in the erect posture do not accord with the fact that the direction of initial head displacement is backwards on the neck, not downwards (Billing and Severin 1959); or with the observation that slipping is commoner on the left side, and is unrelated to the angle made by the epiphysial cartilage with the horizontal (Billing 1954).

The known facts of epiphysiolysis-The following relevant observations emerge from the literature and from other sources. 1) Initial displacement of the neck on the head is forwards, not upwards. 2) In the plane in which slipping occurs, the plate-neck angle normally approximates to 90 degrees. 3) If the asymptomatic opposite hips in patients with epiphysiolysis are examined, the mean angle is reduced to 81 degrees. These three observations emerge from the careful work of Billing and Severin, and establish that the head has migrated posteriorly before clinical and radiographic evidence of frank slipping appears. 4) In such asymptomatic 
opposite hips the cartilage plate is not widened (Burrows 1957). 5) Biopsy findings are those of frank metaphysial fracture, with necrosis of bone causing apparent plate widening radiologically, and attempts at healing (Sutro 1935, Howorth 1949). The foci of chondrofibrous metaplasia reported in two late cases by Lacroix and Verbrugge (1951) do not differ from the same fibrous scarring constantly present in incomplete shear fractures through the tibial apophysis (Uhry 1944). They do not indicate a defect of chondro-osseous maturation as has been assumed. No acceptable evidence for such a defect is revealed by these biopsies. 6) Biopsy findings in early cases reveal little evidence of abnormality (Kleinberg and Buchman 1936). They show no fibrous metaplasia, and no apparent loss of cartilage-bone continuity.

These histological findings tally with the radiological evidence of Billing and Severin. If they are correct, they lead inevitably to the conclusion that epiphysiolysis is a two-stage process, as suggested by Howorth in 1949. The pre-fracture stage-The head has migrated posteriorly on the neck, without loss of cartilage-bone continuity. The only evidence is a plateneck angle reduced below 90 degrees. Symptoms are absent and the plate is radiologically and

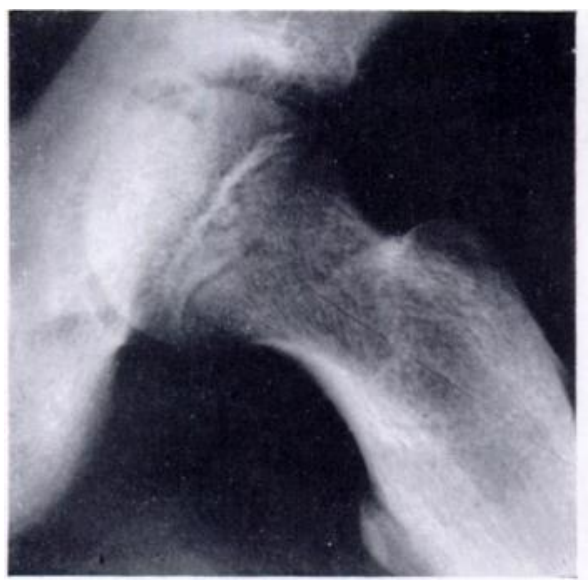

Fig. 2

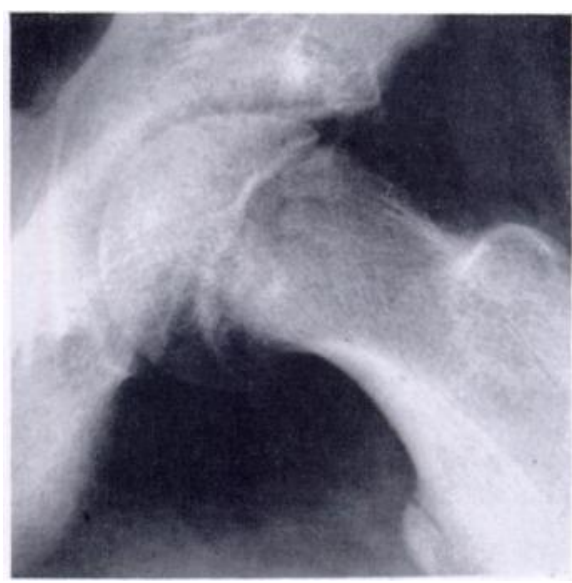

FIG. 3

Epiphysial slipping, before and after fracture. Figure 2-Radiograph of left hip of girl of 13 years before the onset of symptoms. There is no widening of the junctional zone nor evidence of modelling. The epiphysial angle is, however, reduced to 77 degrees indicating that considerable head migration has occurred. Figure 3-The same hip. nine months later. after onset of symptoms. There is frank slipping with widening of the " plate " after fracture, remodelling, and an epiphysial angle of 66 degrees.

histologically normal. The clinically normal hip of a patient with epiphysiolysis is usually in this stage, and commonly will not proceed beyond it. The post-fracture stage-The head now moves backwards much more rapidly, sometimes by sudden or episodic movements. Symptoms are usually present and radiographs show apparent widening of the plate. Biopsy reveals a fracture with secondary absoption of bone, attempts at healing and remodelling. The neck displaces forwards and later upwards and develops further posterior shortening which is now caused by remodelling, as shown by Billing and Severin. Serial radiographs three months apart will show a measurable decrease in the plate-neck angle. An example of these two stages is seen in Figures 2 and 3. 7) While the plate neck angle remains at 90 degrees the shear stresses generated across it in the erect posture are small. Reduction in the angle rapidly increases the shear component, which reaches a value of 50 per cent when the angle is 60 degrees. Fracture at this stage is readily explicable by the normal dynamic stresses on the slanted metaphysis. These stresses do not, however, explain the pre-fracture epiphysial migration, which emerges as the fundamental abnormality. 8) When a growing epiphysial cartilage is exposed to a transverse shear stress, it deviates laterally in response without loss of continuity (Appleton 
1934, Arkin and Katz 1956). It follows that if such a shear force can be identified operating in the appropriate horizontal direction, the growth deviation of the head in Stage I epiphysiolysis can be explained. 9) Epiphysiolysis is commoner on the left side than on the right (Key 1926,

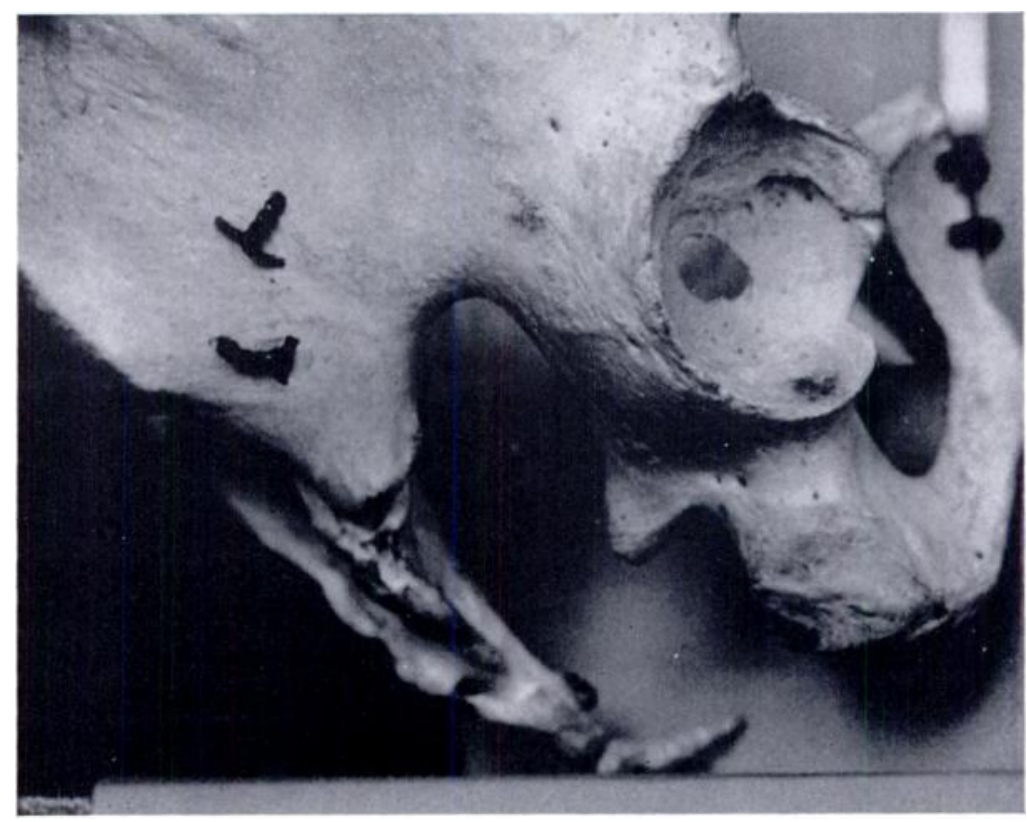

FIG. 4

Photograph of a pelvis in the full slouching position. The acetabular roof is $9 \cdot 5$ centimetres above the seat.

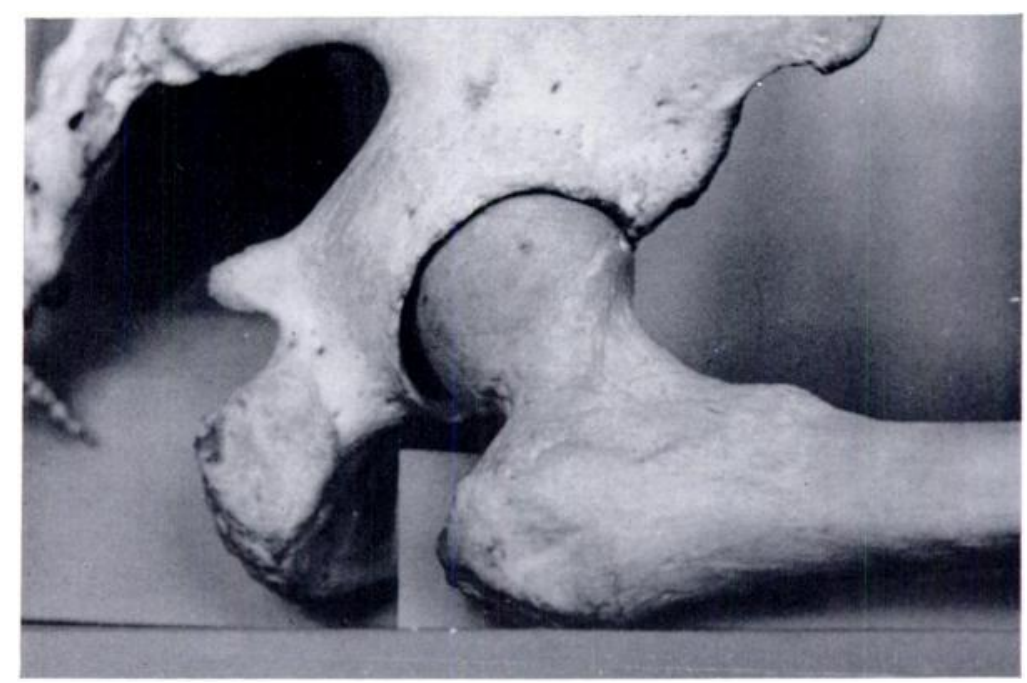

FIG. 5

The pelvis shown in Figure 4, in the writing position. The roof is now 6.8 centimetres above the seat. In this posture an anteverted femur may be surprisingly close to the seat, and in extreme flexion may take most of the weight as the pelvic rotation lifts the ischia off the base of support.

Brailsford 1953, Burrows 1957, Howorth 1957, Theander 1962). The writer found no left predominance in fifty cases analysed locally, but found 430 other cases reported in the literature in which the side incidence was stated. Of the total 480 cases, 292 slipped on the left side and

vOL. 48 B, NO. 2, MAY 1966 


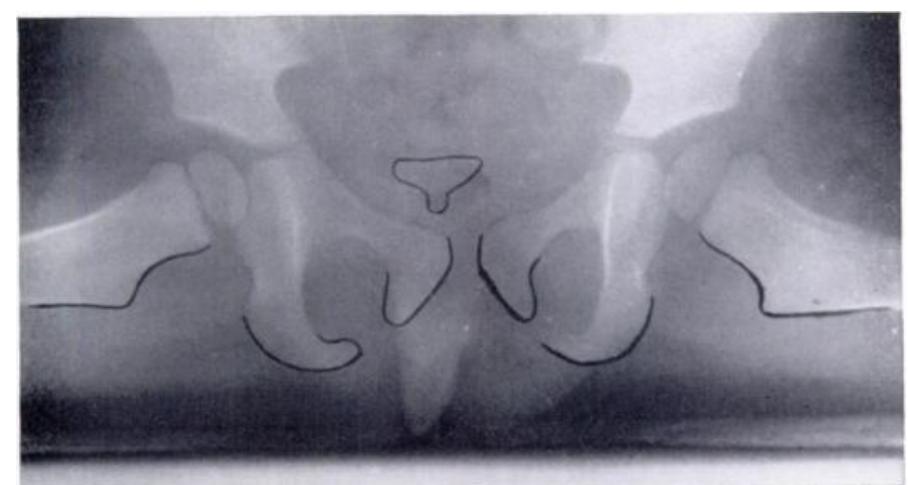

FiG. 6

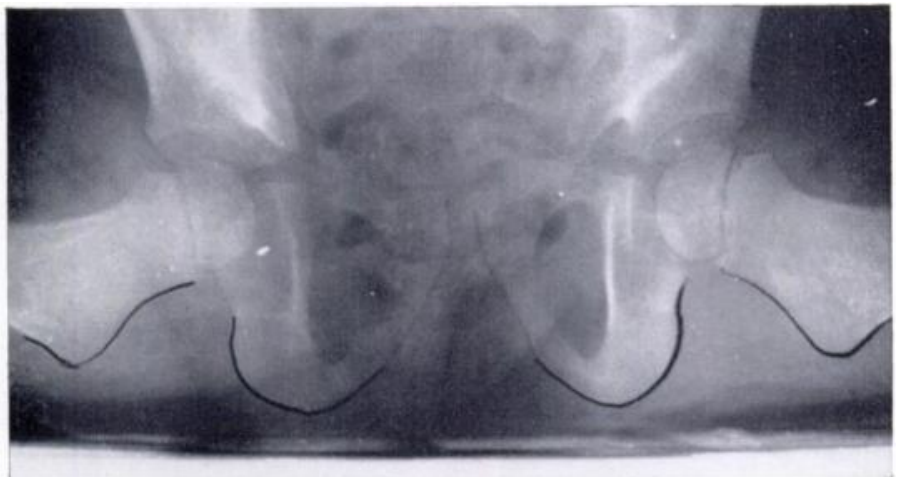

Fig. 7

Sitting radiographs (retouched) of female children aged 21 months and 3 years, showing the relative distances of ischia and trochanters from the seat. One child is sitting erect and the other somewhat flexed, but in both the soft tissue compression extends well out under the trochanters.

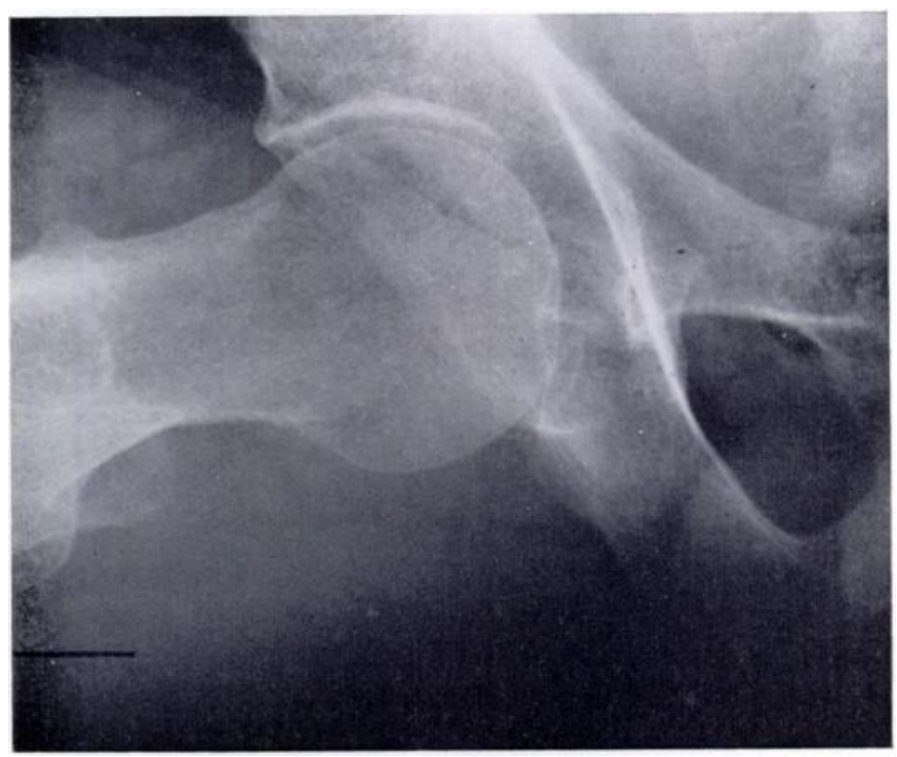

FIG. 8

Sitting radiograph of an adult, in abduction and neutral position. The trochanter is less than 2 centimetres above the plane of the ischia.

THE JOURNAL OF BONE AND JOINT SURGERY 
188 on the right. This difference is highly significant $\left(x^{2}=22 \cdot 6\right)$. This suggests that the shear stress required should tend to be greater on the left side. 10) No right-left asymmetry has been identified in the standing position (Smith 1953) or in walking. Some sports involve asymmetrical leg action, but a more universal manifestation of asymmetry is seen in the tilted body position usual when a seated child is writing.

Statement of hypothesis-From these observations the following hypothesis emerged: 1) The fundamental abnormality of epiphysiolysis is a posterior growth-migration of the head on the neck, unrelated to the erect posture, or to any abnormality of the growth cartilage; and 2) this migration is caused by the shear stress generated while sitting.

Examination of sitting stresses-The literature relating to the sitting posture is scanty. The sitting positions were defined by Strasser (1913), and Akerblom (1948) studied the position with reference to chair design. The major texts on kinesiology do not discuss the sitting posture.

The accepted supposition about sitting is that weight is borne by the ischial tuberosities (Keegan 1953, Dempsey 1962). The idea that any significant stress is borne by the femora has been little considered, except by Akerblom, who showed soft-tissue compression under the thighs and deduced from this that some femoral pressure must exist if the seat is too high. There is, nevertheless, good evidence that some of the pressure is borne by the femora, particularly in the forward (writing) sitting position. In this position the acetabulum is significantly lower than in the erect sitting or in the slouching positions. Measurements of acetabular height in a skeleton studied were respectively $6 \cdot 8,8 \cdot 1$ and $9 \cdot 5$ centimetres in the three positions (Figs. 4 and 5). It follows that the writing position brings the femoral neck

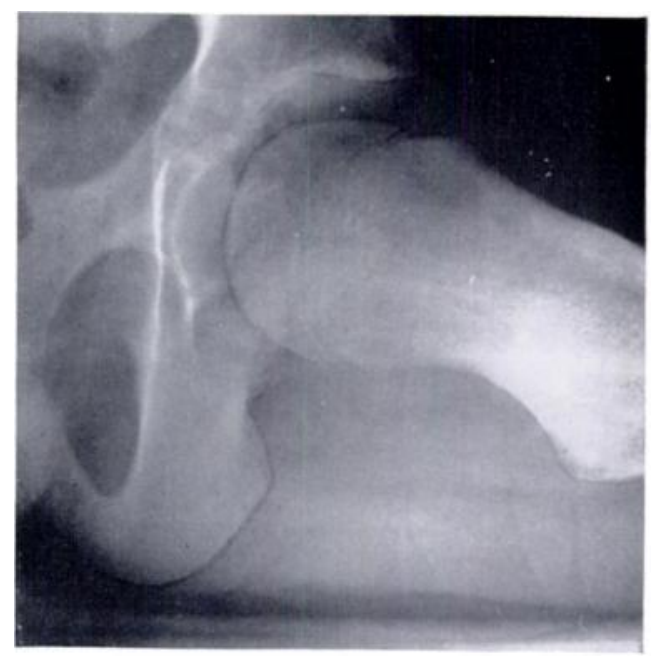

Fig. 9

Sitting radiograph of a boy aged 12 years with slipping epiphysis, showing how the upward sitting stress becomes a forward stress on the femoral neck. closer to the seat than is the case in the other postures. Even when sitting erect, subjects of any age examined radiologically constantly show considerable soft-tissue compression under the femoral necks, indicating the existence of a local compression stress (Figs. 6 to 8 ). The radiographs show, moreover, that the gravity stress acts in such a direction as to displace the neck forwards on the head, as occurs in slipped epiphysis (Fig. 9).

In view of these findings, a quantitative study of sitting stresses was undertaken.

\section{MATERIAL AND METHOD}

The subjects tested were two boys aged nine and eleven, weight 56 pounds ( 25.4 kilograms) and 62 pounds ( 28.2 kilograms) respectively. Two pieces of equipment were used. The first was a buttock-scope made from the design of Dempsey (1962). It consisted of a plate of clear plastic 14 inches by 8 inches by half an inch ( 35 centimetres by 25 centimetres by 12 millimetres) set transversely across the frame of a kitchen chair, edge-lighted and covered with a sheet of rubber dam. The test subjects sat on this in the required position, the foot weight being checked by kitchen scales and the image viewed directly or by mirror from below the plastic. By this device the buttock outlines could readily be traced and ischial tuberosities easily localised within these outlines. The test subject was examined close to a wall and with a strong lateral light so that his shadow could be marked on the wall, and maintained in position. The completed

vOL. 48 B, NO. 2, MAY 1966 


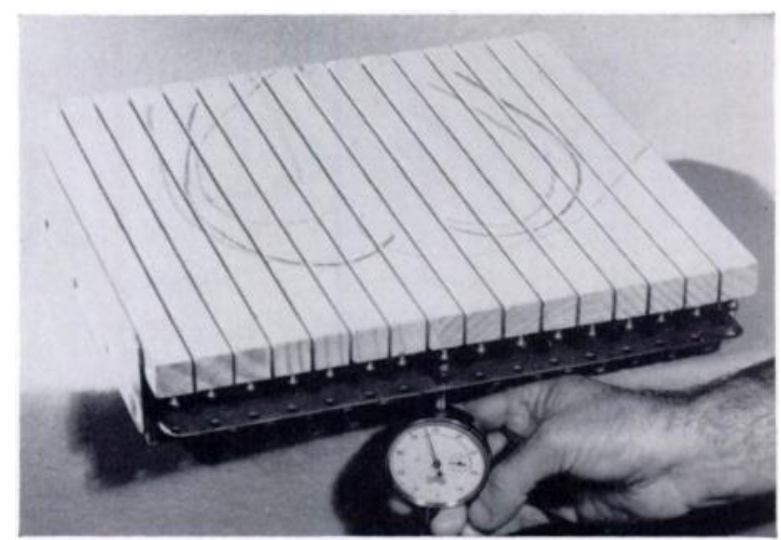

Fig. 10

The buttock-meter, showing the technique of measuring the height of the wooden slats above the base-plate. The slats are labelled $\mathrm{A}$ to $\mathrm{P}$ from right to left. Three buttock outlines are shown. tracing was then transferred to a buttockmeter placed in the same position as the original buttock-scope.

This meter consisted of a wooden platform 123 inches ( 32 centimetres) square and three-quarters of an inch (19 millimetres) deep, sawn into sixteen bars, each three-quarters of an inch wide (Fig. 10). Beneath the wood was a sheet of sponge rubber one inch $(2 \cdot 5$ centimetres) thick, cut into corresponding three-quarter inch segments below each wooden bar. These segments were divided by plastic spacers and the sponge rubber was firmly attached by glue to the bars above and to a solid wooden frame below. A bolt screwed

into each end of each wooden bar projected downwards, and beneath each row of bolts was a perforated brass strip attached rigidly to the baseboard. The distance between the bolts and the brass strip below could readily be measured by a displacement gauge and the measurements were found to be reproducible with a variation not exceeding three-thousandths of an inch. The buttock-meter was tested and found to obey Hooke's law over the expected range of pressures. Before use it was calibrated, the base-line measurement for each end of each bar being recorded as a datum point.

The buttock outline derived from the scope was traced on to the wooden slats of the buttock-meter. The test subject then sat on the meter, adjusting his position until the buttock outlines corresponded. The position was checked by noting the wall shadow marking and the reading on the scale under the feet. Readings were then taken with the displacement gauge at each end of each bar to determine the depression (or elevation) from the datum reading, the first six readings being repeated at the end of the series to make sure that the subject had not moved appreciably while the measurements were being taken.

The results in each position were represented in diagrammatic form as shown in Figure 11, the position of the greater trochanters being defined in each case by palpation, and the ischial tuberosity positions being derived from the buttock-scope tracing. From the readings at the front and back of each bar, the positions of the resultant force were calculated and this was marked in the diagram for each bar. It can be shown that the magnitude of the resultant force on each bar is represented by the algebraic sum of the deflections, and as the total of all these forces equalled the sitting weight of the subject in the position tested, the gravity force per unit deflection could be calculated. The total gravity force per

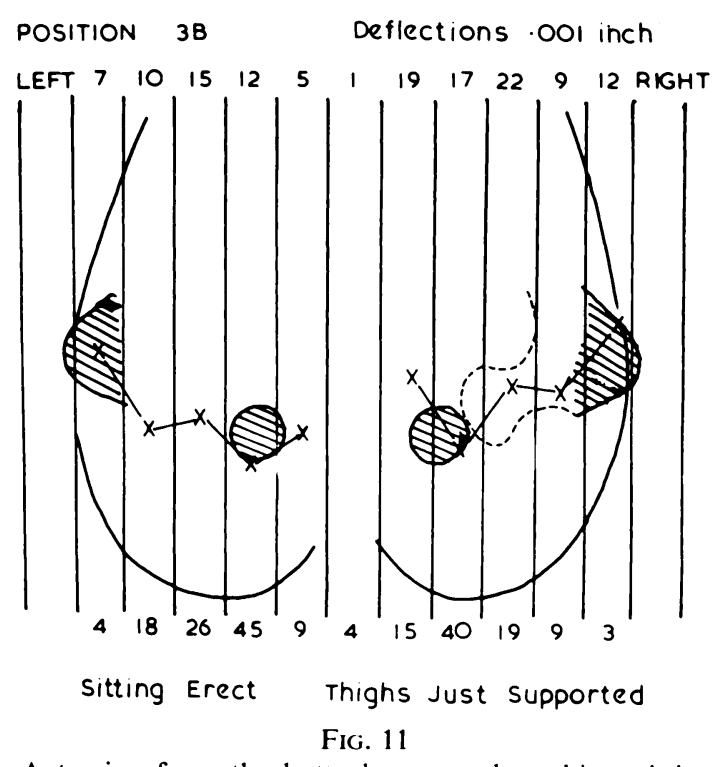

A tracing from the buttock-meter, the subject sitting erect. The circles represent the ischia, located from the buttock-scope. The deflection figures indicate the depression from the base-line which has occurred at the front and back of each slat. The cross on each slat is the calculated locus of the resultant force. 
bar was then recorded as a histogram for each position. Several different positions were tested for each subject. The results in the four positions relevant to this hypothesis are shown in Figures 12 to 15.

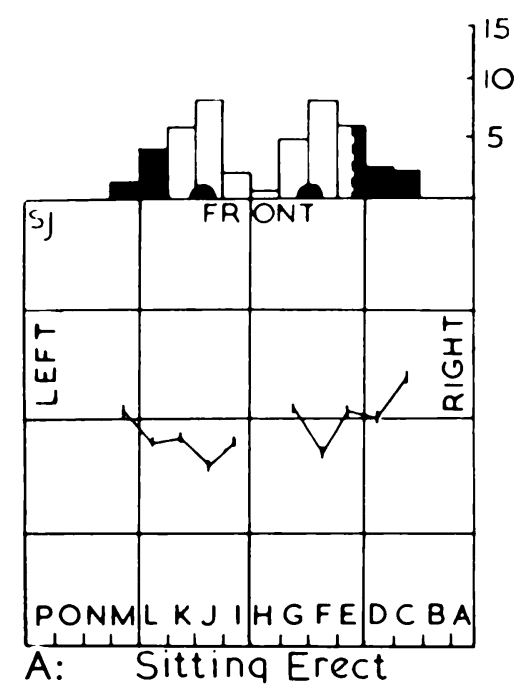

FIG. 12

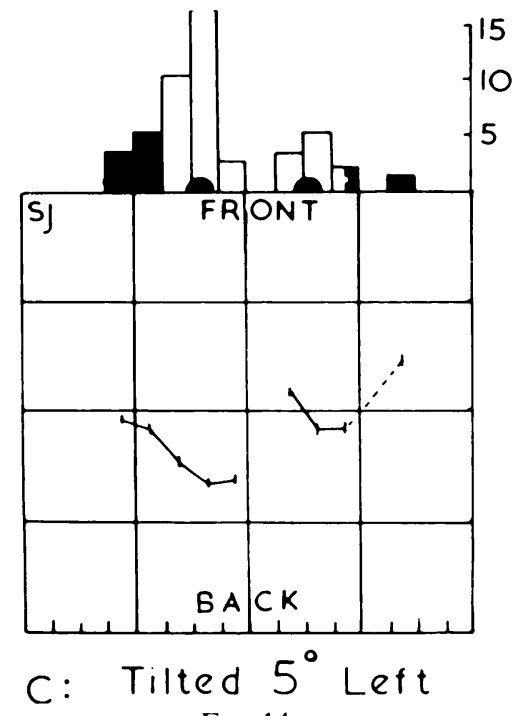

Fig. 14

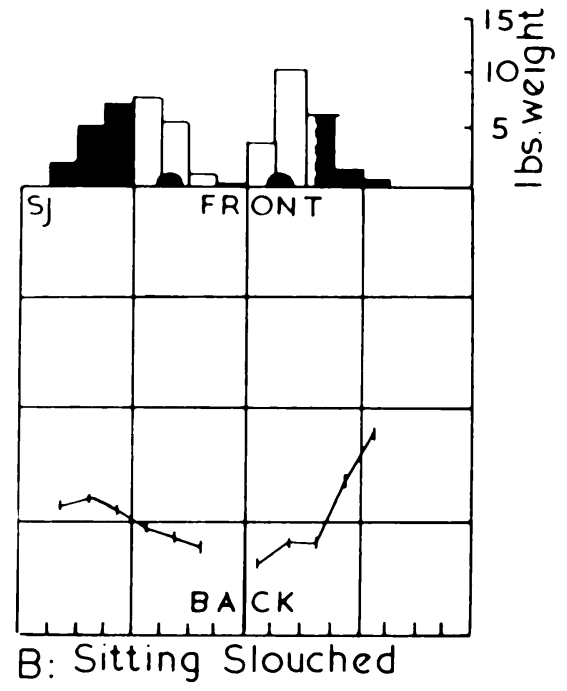

FIG. 13

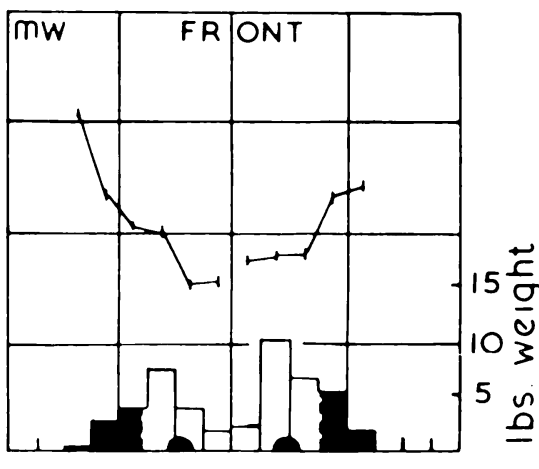

D: Writing, Arm on Desk

FIG. 15

Sitting stress diagrams derived from four different sitting positions. The square represents the buttock-meter surface, and the line graph on it the location of the resultant force on each slat. The histogram shows the force in pounds weight on each slat. The black semi-circles represent the locus of the ischial tuberosities, and the black part of the histogram represents that part of the sitting stress which is exerted on the femora. Figure 12 is derived from the evidence shown in Figure 11.

\section{RESULTS}

In all positions except the cross-legged position, the buttock-meter readings indicated a much wider lateral spread of gravity pressure than has previously been assumed. The idea that almost all the gravity force is borne directly by the ischia is not confirmed by these findings. When the position of the more lateral resultant force is plotted it is seen to correspond reasonably well with the estimated position of the femoral neck when the subject is sitting erect;

VOL. 48 B, NO. 2, MAY 1966 
extending farther down the shaft in the writing, and particularly in the leg-hanging positions. The weight was seldom evenly distributed on the two sides. In the right-handed writing position the gravity force was greater on the left if the left elbow was off the table, but did not show a constant predominance on either side if the elbow was supported on the table.

Figure 16 is a reconstruction from Figure 11 as it would appear in the vertical plane through the right femoral neck, the bony measurements and proportions being derived directly from a skeleton (Fig. 17) and the proportionate spread of the gravity force over the skeleton from Figures 11 and 12. The anteversion angle was set at 20 degrees which is an average value at the age of ten (Billing 1954). The location of the epiphysial cartilage was determined by measuring its location in the cervical axis in the radiographs of six ten-year-old boys and transferring the mean of the values found to the diagram. It has been shown above that in this position the femoral neck plane corresponds well with the plane of the resultant forces; and the

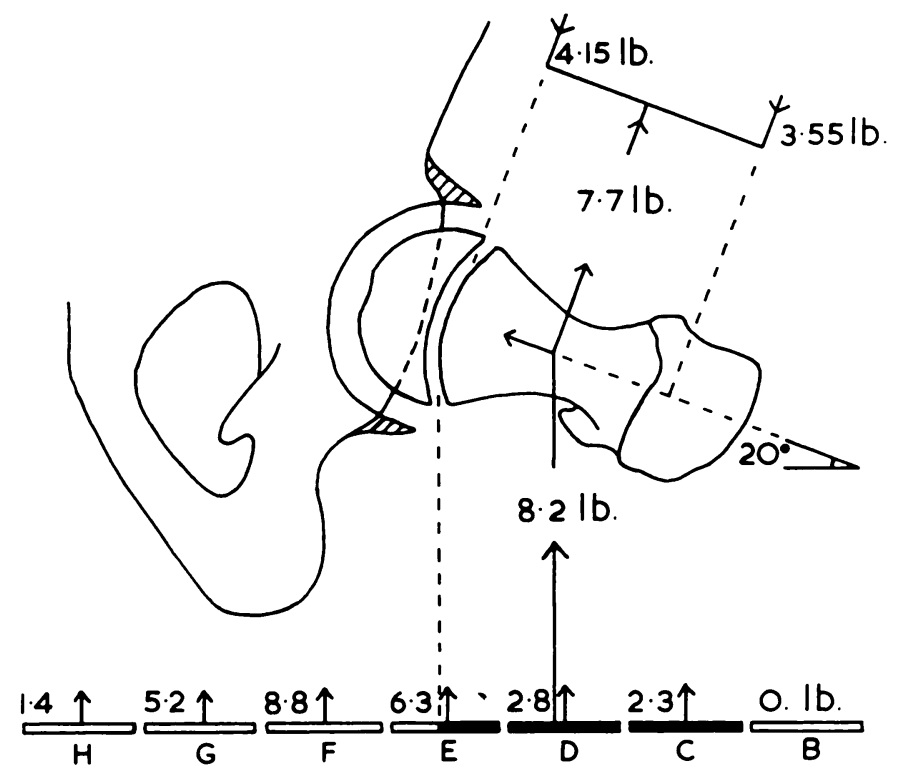

Fig. 16

Diagram to show the estimated relation of the forces shown in Figures 11 and 12, to the skeletal structures of the right hip area. This is based on the known locus of the ischium and trochanter relative to the slats and on the anatomical proportions of the skeleton shown in Figure 17. In this example, there is no slat division close to the plane of the epiphysial plate. The calculations are based on the assumption that half the stress on slat $E$ is exerted on the femur. The force of $8 \cdot 2$ pounds weight is the resultant of the forces exerted on slats $C, D$, and $E$.

reconstruction indicates that in this position a significant proportion of the gravity force-of the order of 30 per cent-is borne by the femur. These forces can be resolved into a single resultant force acting on the neck as shown. In this particular example the resultant force is 8.2 pounds ( 3.7 kilograms) acting on the right femoral neck of a subject weighing 62 pounds (28.2 kilograms). After correction for the anteversion angle it can be deduced that this force is opposed by a torsional force of 3.6 pounds ( 1.6 kilograms) weight in the femoral shaft, due to the resistance to rotation at the knee joint, and by a shear force of $5 \cdot 15$ pounds $(2 \cdot 3$ kilograms) weight at the acetabulum. This shear is acting in the required direction to cause anterior growth deviation of the neck on the head. If it is assumed that this stress will increase proportionately with weight, the corrected value for a child weighing 100 pounds $(45.5$ kilograms) will be 6.7 pounds ( 3 kilograms) weight. 
Observation suggests that the average schoolchild spends about ten hours a day sitting down, as opposed to three standing up. On this basis the deviating effect of the sitting force will be 67 pound-hours, (30.5 kilogram-hours) for the hypothetical 100-pound subject. Estimates of the effect of the horizontally acting forces in the erect posture are necessarily approximate but yield a value of less than half this amount.

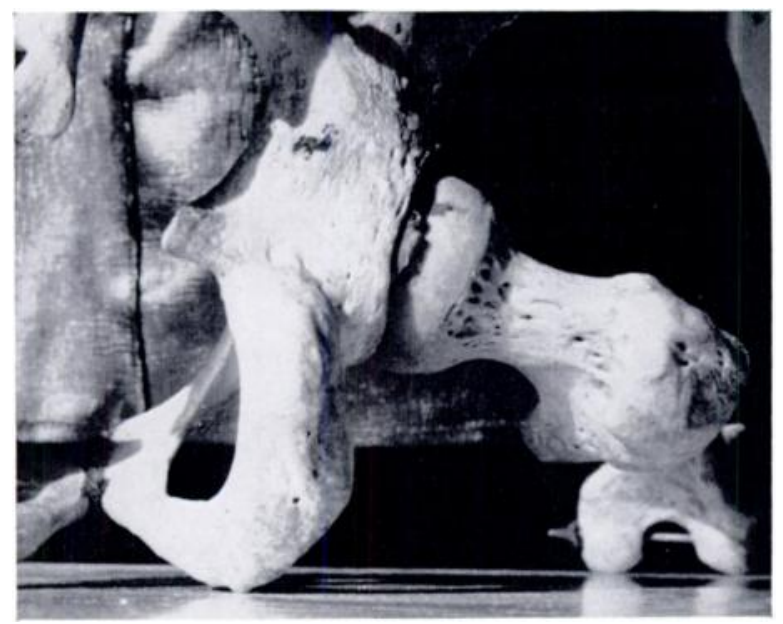

FIG. 17

Photographs of a pelvis and femur, placed in the 'upright' sitting position.

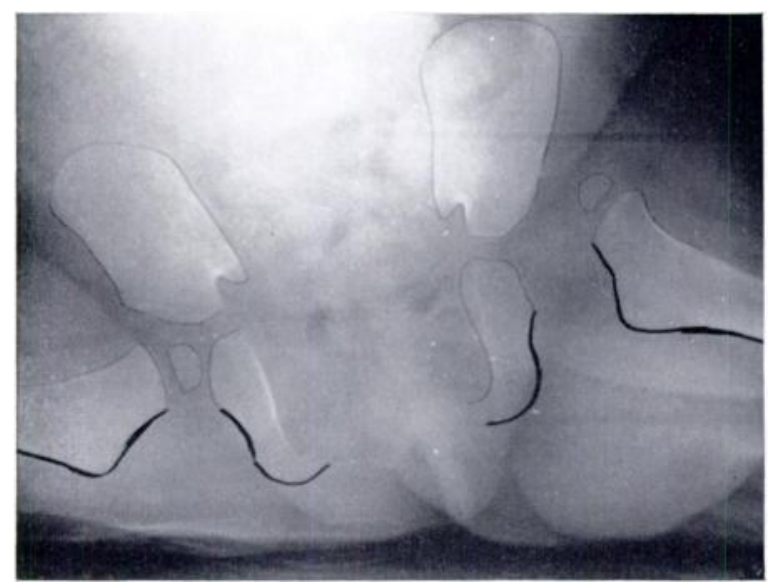

FIG. 18

Sitting radiograph of a child with congenital dislocation of the right hip: aged 7 months. The child is tilted 18 degrees to the left, and the left trochanter is closer to the seat than the right ischium. There is still sufficient pressure on the right to dislocate the femoral head anteriorly.

Conclusions-It is concluded that the sitting position results in a significant shearing stress acting from behind forwards across the femoral neck in the manner postulated as necessary to explain the posterior growth deviation of the head.

This force will vary in magnitude with different anatomical characteristics and in different sitting positions, tending to be greatest in the writing position.

Sitting stresses in the writing position-The findings in the buttock-meter investigation suggested that writing might produce excessive left-sided stress under certain conditions, and an investigation of sitting habits and stresses was therefore undertaken.

vol. 48 B, NO. 2, MAY 1966 
Radiographs in the lateral-tilted sitting position show that pelvic asymmetry causes an approximation of the femur on the down-tilted side and elevation of the opposite ischium (Fig. 18). Observations of classes writing in school revealed a constant tendency for righthanded children to tilt the body axis to the left and for left-handed ones to tilt it to the right. Figures 19 and 20 illustrate the asymmetrical position adopted by three school children, two right-handed and one left-handed, during a four-minute period of writing. The extra weight resulting from the tilting is compensated by supporting the non-writing forearm or elbow on the desk.

To test the effect of this support the two right-handed subjects were tested for lateral deviation of their sitting stresses while writing. Each sat in turn on a seat having vertical boards as its lateral supports. A pair of bathroom scales was placed between the seat and the floor in such a manner that the vertical support on each side rested in the centre of the scale platform. In this position the initial scale readings were identical. The test subjects then sat on

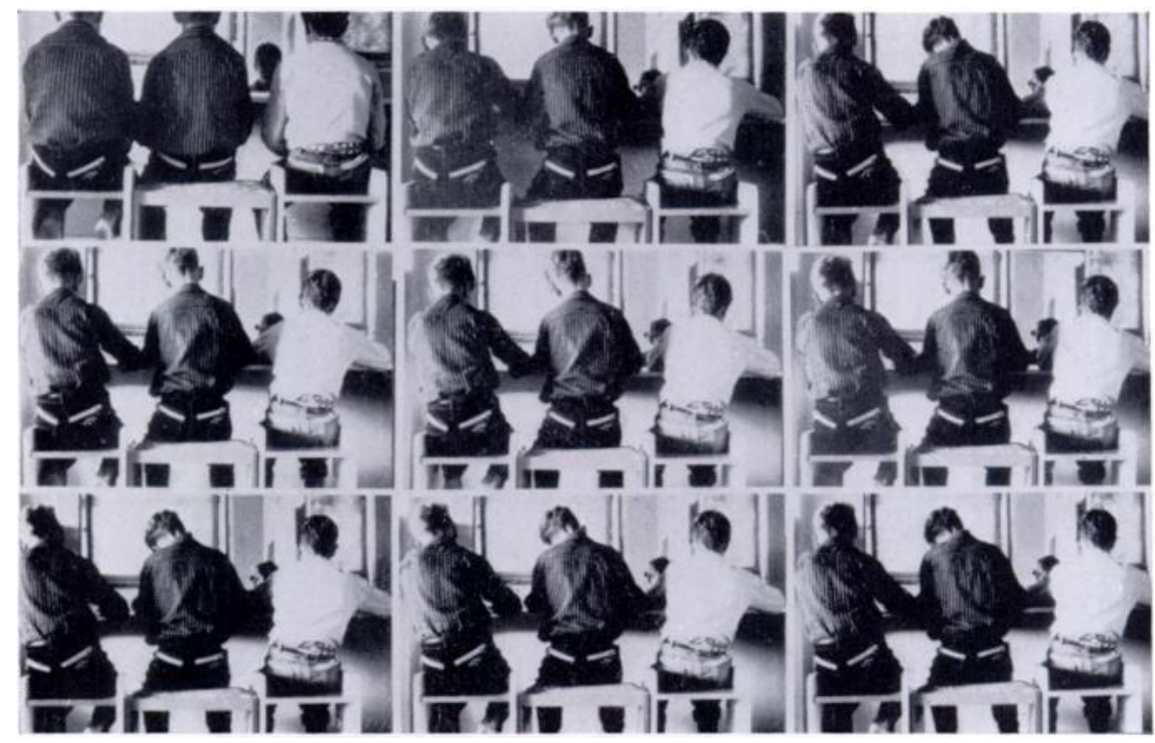

FIG. 19

Composite photograph of three children sitting at a desk. In the top left-hand picture the children were reading. They were then instructed to start writing, and the subsequent photographs were taken at thirty-second intervals. The boy in the white shirt is left handed, the other two right handed.

the seat and simultaneous readings were taken from each scale at twenty-second intervals. The subjects were tested in three positions-sitting erect reading, writing with the left elbow on the desk, and writing with the left forearm on the desk, but the elbow unsupported. Sixteen readings were taken of each subject in each position and the results are shown in Table I.

In the reading and elbow support writing positions, no significant difference between the two sides was detected. In the second writing position both children showed a greater mean reading on the left side, and in both this difference is significant $(t=4.4 ; \mathrm{P}<\cdot 001 ; \mathrm{P}<\cdot 001)$.

It is concluded that in the writing position the gravity axis is deviated away from the side of the writing arm, and if the extra force thus generated on this side is not taken by the elbow of the non-writing arm, it will manifest itself as a relative increase in buttock pressure on this side.

It follows that if sitting stress is the basic cause for slipped epiphysis the position does incorporate a reasonable explanation for the left-sided predominance found.

Additional evidence-While these results were being analysed, a monograph on the sitting position was published by Schoberth (1962). Schoberth also designed a buttock-meter; he 
examined twenty-eight patients. He found that the area of support was much greater than that contained between the ischial tuberosities. He was able to show that in the writing position pressure was exerted on the thighs and upper femora. Of the twenty-eight patients examined in the writing position, seventeen showed a heavier loading on the left side and six on the right

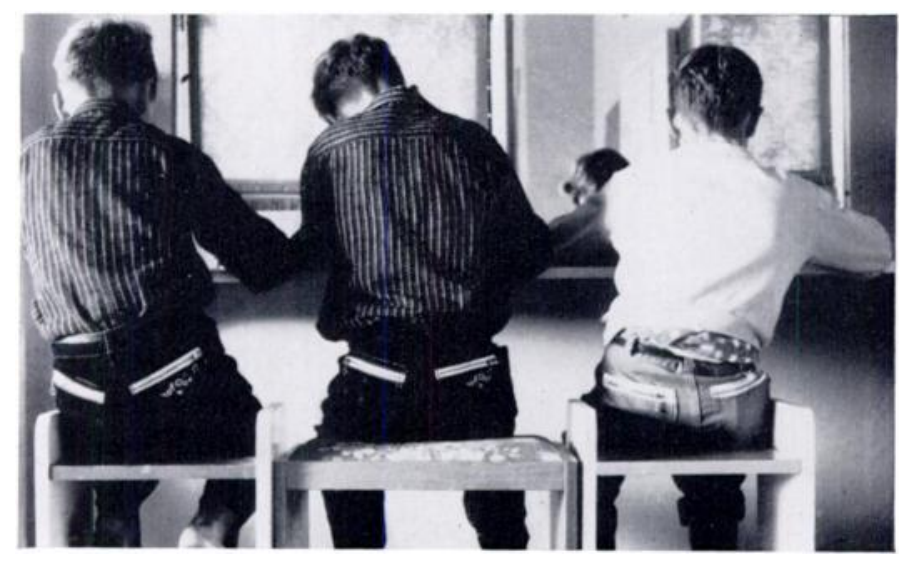

FIG. 20

Enlargement of the last panel from Figure 19.

side. This difference is significant $\left(x^{2}=5 \cdot 2\right)$ and Schoberth concluded that it was due to righthandedness. The two investigations are thus in all respects confirmatory and are in accordance with the hypothesis being tested.

If this sitting force during growth in fact exists, it should operate to reduce the anteversion angle of white races below that found at birth and in adult primitives. That this reduction

TABLE I

Mean Gravity Pressures (Pounds) Found in the Sitting Position

\begin{tabular}{|c|c|c|c|c|}
\hline \multirow{2}{*}{ Position } & \multicolumn{2}{|c|}{ Subject 1} & \multicolumn{2}{|c|}{ Subject 2} \\
\hline & Right & Left & Right & Left \\
\hline Reading & $25 \cdot 7$ & $25 \cdot 7$ & $28 \cdot 0$ & $28 \cdot 3$ \\
\hline Writing, left elbow on the desk & $25 \cdot 0$ & $26 \cdot 4$ & $26 \cdot 1$ & $25 \cdot 1$ \\
\hline Writing, left elbow off the desk & $25 \cdot 1$ & $28 \cdot 3$ & $25 \cdot 1$ & $34 \cdot 5$ \\
\hline
\end{tabular}

during growth occurs is well attested (Kingsley and Olmsted 1948); and the finding that Polynesians have a high mean angle of 27 degrees supports the latter suggestion.

Further support derives from the age incidence of epiphysiolysis. The peak growth rate in adolescence is at $11_{4}^{3}$ years for girls and 13! years for boys (Mackenzie, Court Brown, Doll and Sissons 1961), after which the rate drops rapidly. As the rate of growth deviation will be a function of the growth rate it should produce a peak incidence of slipping close to this growth rate peak. This tallies with the mean age incidence derived from the 480 cases examined above -11.4 years for girls and 14 years for boys.

The two to one male predominance is a predictable consequence of the more rapid peak growth rate and longer growth period of the male. Similarly, the rapidly growing eunuchoid type of child would be vulnerable as a consequence of his growth rate, and the overweight sedentary child as a consequence of his increased static shear stress. This incorporates Harris's 
endocrine imbalance as a predisposing factor, but suggests that it is not necessary to invoke a direct fragility effect to explain the frequent occurrence of slipping in these "endocrine" children.

\section{DISCUSSION}

The concept of chair sitting as a mechanically undesirable position capable of causing deformity is at first sight surprising. Viewed against man's evolutionary background it becomes reasonable.

Man appears to be about three-quarters of a million years old as a genus. For about 99 per cent of this time he has been a hunting, food-gathering nomad. The agrarian transition which made permanent settlement feasible occurred less than 10,000 years ago (Braidwood 1960), and only then could the chair replace the transient and uncomfortable squatting postures of the nomad.

In fact the practice of prolonged chair sitting is one of the most important and dramatic changes in man's environment since the discovery of agriculture. He has had no time at all to adapt to this revolutionary practice, and it would be surprising if such a habit change were harmless.

\section{SUMMARY}

1. The hypothesis is advanced that the fundamental lesion of epiphysiolysis is a slow posterior growth migration of the head on the neck.

2. It is suggested that this is unrelated to the erect posture or to any abnormality of the growth cartilage.

3. Sitting stresses are measured and are postulated as the likely cause of the growth deviation.

4. The result of the deviation is an increase in the shear stress component; in the erect posture clinical epiphysiolysis is regarded as a simple fracture occurring in a proportion of deviated cases when the increased shear component exceeds the critical level appropriate to the individual. 5. It is suggested that this hypothesis explains the age and sex incidence, the left predominance, the reduced epiphysial angle found on the uninvolved hip and the clinical and radiological evolution of the disorder.

It is a pleasure to acknowledge generous help received from the Auckland Medical Research Foundation, the Auckland Hospital Board and the Department of Scientific and Industrial Research. I am also indebted to Drs R. T. Marshall and P. Wrightson of Auckland Hospital, to the staffs of the Auckland Hospital Photographic and Statistical Departments and of the Marion Davis Memorial Library. I would like particularly to thank Mr W. R. Beasley of the Department of Scientific and Industrial Research whose help made the project possible, and it is not out of place to acknowledge that the hypothesis proposed stems directly from the prior researches of Billing and of Arkin and Katz.

\section{REFERENCES}

Akerblom, B. (1948): Standing and Sitting Posture. Stockholm: A. B. Nordiska Bokhandeln.

ANDRÉn, L. and Borgström, K. E. (1958): Seasonal Variation of Epiphysiolysis of the Hip and Possibility of Causal Factor. Acta Orthopaedica Scandinavica, 28, 22.

Appleton, A. B. (1934): Postural Deformities and Bone Growth. Lancet, i, 451.

Arkin, A. M., and KATZ, J. F. (1956): The Effects of Pressure on Epiphyseal Growth: The Mechanism of Plasticity of Growing Bone. Journal of Bone and Joint Surgery, 38-A, 1056.

Backman, S. (1957): The Proximal End of the Femur. Acta Radiologica, Supplementum 146.

Billing, L. (1954): Roentgen Examination of the Proximal Femur End in Children and Adolescents. Acta Radiologica, Supplementum 110.

Billing, L., and Severin, E. (1959): Slipping Epiphysis of the Hip. Acta Radiologica, Supplementum 174.

Braidwood, R. J. (1960): The Agricultural Revolution. Scientific American, 203, 130.

BraILSFORD, J. F. (1953): The Radiology of Bones and Joints. Fifth edition. London: J. \& A. Churchill Ltd.

Burrows, H. J. (1957): Slipped Upper Femoral Epiphysis. Journal of Bone and Joint Surgery, 39-B, 641.

Dempsey, C. A. (1962): Body Support/Restraint. Product Engineering, 33, 8, 106.

Dulligan, P. J., Jun. (1953): The Etiology of Slipping of the Capital Femoral Epiphysis. New York State Journal of Medicine, 53, 2643.

HaRris, W. R. (1950): The Endocrine Basis for Slipping of the Upper Femoral Epiphysis. An Experimental Study. Journal of Bone and Joint Surgery, 32-B, 5. 
Howorth, M. B. (1941): Slipping of the Upper Femoral Epiphysis. Surgery, Gynecology and Obstetrics, 73, 723. HowORTH, M. B. (1949): Slipping of the Upper Femoral Epiphysis. Journal of Bone and Joint Surgery, 31-A, 734. Howorth, M. B. (1952): A Textbook of Orthopedics. Philadelphia and London: W. B. Saunders \& Co. Howorth, M. B. (1957): Slipping of the Upper Femoral Epiphysis. Clinical Orthopaedics, 10, 148.

KeEgan, J. J. (1953): Alterations of the Lumbar Curve Related to Posture and Seating. Journal of Bone and Joint Surgery, 35-A, 589.

KeY, J. A. (1926): Epiphyseal Coxa Vara or Displacement of the Capital Epiphysis of the Femur of Adolescence. Journal of Bone and Joint Surgery, 8, 53.

Kingsley, P. C., and Olmsted, K. L. (1948): A Study to Determine the Angle of Anteversion of the Neck of the Femur. Journal of Bone and Joint Surgery, 30-A, 745.

Kleinberg, S., and Buchman, J. (1936): The Operative Versus the Manipulative Treatment of Slipped Femoral Epiphysis. With a Description of a Curative Operation. Journal of the American Medical Association, 107, 1545.

Lacroix, P., and Verbrugge, J. (1951): Slipping of the Upper Femoral Epiphysis. A Pathological Study. Journal of Bone and Joint Surgery, 33-A, 371.

LöfGren, L. (1953): Slipping of the Upper Femoral Epiphysis, Signs of Endocrine Disturbance, Size of Sella Turcia, and Two Illustrative Cases of Simultaneous Slipping of the Upper Femoral Epiphysis and Tumour of the Hypophysis. Acta Chirurgica Scandinavica, 106, 153.

Mackenzie, A., Court Brown, W. M., Doll, R., and Sissons, H. A. (1961): Mortality from Primary Tumours of Bone in England and Wales. British Medical Journal, i, 1782.

Michele, A. A. (1960): Development of Anomalies in Man: Iliopsoatic Syndromes. Journal of Bone and Joint Surgery, 42-A, 901.

PonSETI, I. V. (1956): Legg-Perthes Disease. Observations on Pathological Changes in Two Cases. Journal of Bone and Joint Surgery, 38-A, 739.

Ponseti, I. V. (1957): Skeletal Lesions Produced by Aminonitriles. Clinical Orthopaedics, 9, 131.

Ponseti, I. V., and MCClintock, R. (1956): The Pathology of Slipping of the Upper Femoral Epiphysis. Journal of Bone and Joint Surgery, 38-A, 71.

Rennie, A. M. (1960): The Pathology of Slipped Upper Femoral Epiphysis. A New Concept. Journal of Bone and Joint Surgery, 42-B, 273.

SChoberth, H. (1962): Sitzhaltung, Sitzschaden, Sitzmöbel. Berlin: Springer-Verlag.

Selye, H., and Ventura, J. (1957): Effect of Hypophysectomy and Substitution Therapy With STH upon Experimental Bone Lathyrism. American Journal of Pathology, 33, 919.

Smith, J. W. (1953): The Act of Standing. Acta Orthopaedica Scandinarica, 23, 159.

STrASSER, H. (1913): Quoted by Schoberth.

Sutro, C. J. (1935): Slipping of the Capital Epiphysis of Femur in Adolescence. Archives of Surgery, $31,345$.

Theander, G. (1962): Pelvic Instability in Upper Femoral Epiphysiolysis. Acta Orthopaedica Scandinavica, 32 , 52.

UhrY, E. (1944): Osgood-Schlatter Disease. Archives of Surgery, 48, 406.

Waldenström, H. (1940): Slipping of the Upper Femoral Epiphy'sis. Surgery, Gynecology and Obstetrics, 71, 198. 